patients admitted with HF (improved coding, patient identification, increased use of disease modifying medications in HFREF and better outpatient follow up and support in the community). It is also encouraging to observe a reduction in re-admission rates and 30 day mortality. This is interesting in that we now code a significant number of additional patients with severe valvar heart disease as having HFPEF and these patients are at considerable risk of early readmission and death. Over the next year we plan to continue to develop the IHFS particularly with improved education and self-management of HFPEF patients, cardiac rehabilitation programmes, and validation of GP HF registers.

\section{VENOUS CONGESTION AS MEASURED BY ECHOCARDIOGRAPHY PREDICTS SEVERITY OF RENAL DYSFUNCTION AND SURVIVAL IN PATIENTS WITH HEART FAILURE}

Jagdeep Singh* ${ }^{*}$ AH Muhammad Iqbal, Bayan Soujeri, Daniel Levin, Chim C Lang. University of Dundee; *Presenting Author

\subsection{6/heartjnl-2016-309890.7}

Introduction Renal dysfunction in patients with heart failure (HF) has traditionally been attributed to poor cardiac output. There is currently a growing body of evidence to suggest that renal venous congestion (VC) plays a more important role than hypo-perfusion. However, a vast majority of them have been invasive studies measuring pulmonary artery and central venous pressures as markers of venous congestion. We, therefore, aimed to determine if $\mathrm{VC}$ as determined by inferior vena cava (IVC) dilatation using echocardiography was associated with worsening renal function, HF hospitalisation and all-cause mortality.

Methods We designed a population-based, longitudinal cohort study of 1034 unselected (acute/chronic) HF patients. All patients were symptomatic and required the use of loop diuretic therapy. VC was defined as IVC diameter $>2.1 \mathrm{~cm}$ as determined by echocardiography performed nearest to the time of recruitment into study. Renal function was determined by estimated glomerular filtration rate (eGFR) using the abbreviated MDRD equation. Logistic regression models were used to examine the association between VC and eGFR. Cox proportional hazard models were applied to examine the influence of VC on all-cause mortality and CHF hospitalisations.

Results Logistic regression models showed that those with severe renal impairment $(\mathrm{eGFR}<30)$ were more likely to have VC compared to those with an eGFR $>60$ (Odds Ratio=7.7; 95\% CI(1.6-10.5), $\mathrm{p}=0.012)$. Multivariate analysis showed that those with VC had significantly worse survival than those without VC after adjusting for age, sex, eGFR and furosemide daily dose (hazard ratio [HR]: 1.6, 95\% CI: 1.15-1.96; p = 0.002). There was also a nonsignificant trend towards shorter time to first hospitalisation for $\mathrm{HF}$ in the VC group compared to those without VC (hazard ratio [HR]: 1.22, 95\% CI: $0.96-$ $1.56 ; \mathrm{p}=0.103)$.

Conclusions VC as determined by dilated IVC on echocardiography is associated with worsening renal function in an unselected group of HF patients. This readily available and noninvasive test can also be used to predict HF hospitalisation and all-cause death in this patient group.
8 HEPATIC FUNCTION PREDICTS HOSPITALISATION AND ALL CAUSE DEATH IN PATIENTS WITH HEART FAILURE

Jagdeep Singh*, Daniel Levin, Chim C Lang. University of Dundee; *Presenting Author

\subsection{6/heartjnl-2016-309890.8}

Introduction Hepatic dysfunction has always been considered as a sequel of chronic heart failure (CHF), however there has been recent interest in it being used as a marker of disease severity instead. Previous studies have demonstrated this among patients in clinical trial settings but no population based studies have been conducted thus far. We aim to determine the impact of hepatic dysfunction on a composite of all cause mortality and heart failure hospitalisation in patients treated for CHF.

Methods We analysed data from the Systems BIOlogy Study to Tailored Treatment in Chronic Heart Failure (BIOSTAT$\mathrm{CHF}$ ) database which prospectively tracks treatment, comorbidity, blood investigations, hospitalisation and death information of patients with heart failure from Tayside and Fife. Cox proportional hazard models were used to assess the prognostic impact of liver dysfunction on heart failure outcomes, while controlling for covariates like treatment regime, previous history of myocardial infarctions, atrial fibrillation, renal disease and CHF duration.

Results Out of a total 1805 patients, there were 1200 $(66.5 \%)$ males, with a mean age of $73.6( \pm 10.7)$ years, and mean duration of HF 39.9 months with a total of 414 all cause death or heart failure hospitalisation. We found low serum albumin levels (less than $35 \mathrm{~g} / \mathrm{L}$ ) to be an independent predictor of $\mathrm{CV}$ death or hospitalisation with a hazard ratio of 2.05 (95\% CI 1.51-2.79, p $<0.001$ ). Similarly, elevated bilirubin (more than $20 \mu \mathrm{mol} / \mathrm{L}$ ) and alanine aminotransaminase (ALT) (more than $35 \mathrm{U} / \mathrm{L}$ ) increased the risk of outcomes with hazards of 1.97 (95\% CI 1.56-2.50, p < 0.001) and 1.31 (95\% CI 1.04-1.66, $\mathrm{p}=0.024)$ respectively.

Conclusions Our findings demonstrate lower serum albumin, elevated total bilirubin and ALT were independent predictors of all cause death or hospitalisation among an unselected group of HF patients. These markers can be easily used to risk stratify ambulatory CHF patients in the clinic.

\section{ARE WE READY FOR OUTPATIENT ACUTE HEART FAILURE MANAGEMENT (FRUSEMIDE LOUNGES AND BEYOND)?-A NATIONWIDE SURVEY OF UK ACUTE HEART FAILURE PRACTICE}

Kevin Mohee*, Kenneth Wong. Academic Department of Cardiology; *Presenting Author

\subsection{6/heartjnl-2016-309890.9}

Purpose Heart failure (HF) has a prevalence of over 750,000 people in the UK and over 23 million worldwide. In response to the burden of hospital readmissions and post hospitalisation needs of HF patients, recent health policies stress the need to develop services that will cater for patients' requirements and deliver these closer to their home. One such service is out of hospital parenteral diuretic treatment, which is supported by data from observation studies although there may be observation bias. We aimed at carrying out an online survey to evaluate use of outpatient acute HF management in the UK and assessing the feasibility of setting up a randomised control trial 
(RCT) comparing inpatient versus outpatient acute heart failure management in the UK.

Method We developed a brief online survey by using SurveyMonkey ${ }^{\circledR}$ (Palo Alto, Calif) that was sent to 237 consultant cardiologists with an interest in heart failure in the UK identified from the Directory of Cardiology 2014. The survey was available from 26 February through 10 March 2015 and comprised questions regarding existence of an outpatient acute heart failure service (frusemide lounge) at the hospital where the consultant currently works, whether hospital/PCT currently support a community based acute heart failure management service including the use of parenteral frusemide, identity of person who delivers the community parenteral frusemide service, interest to take part in a multi-centre randomised controlled trial comparing outpatient acute heart failure management with standard inpatient care and if so the number of patients that can potentially be randomised over the next 2 years.

Results The survey was sent by direct e-mail invitation to 237 cardiologists; 55 (23\%) took and completed the survey. 14 $(25 \%)$ indicated existence of an outpatient acute heart failure service (frusemide lounge) at the hospital where they currently work and $14(25 \%)$ mentioned that their hospital/PCT currently support a community based acute heart failure management service including the use of parenteral frusemide. Of these $14(25 \%)$ centres, in $5(9 \%)$ delivery of the community parenteral frusemide service was provided by district nurses, 2 $(3.5 \%)$ by GP, $7(12.5 \%)$ by heart failure nurse and $1(1.7 \%)$ by an ambulatory care unit. Finally 21 (37.5\%) expressed an interest in taking part in a multi-centre randomised controlled trial comparing outpatient acute heart failure management with standard inpatient care.

Conclusion Our study shows that only a very small minority of hospitals in the UK offers an outpatient based acute heart failure management such as frusemide lounges. No RCT has investigated the clinical effectiveness and safety of outpatient IV diuretic therapy in patients with decompensated HF yet. Hence our aim is to carry out the first RCT looking at safety and effectiveness of Out-of-hospital Acute Heart failure Care compared with inpatient management in the UK.

\section{PSYCHIATRIC CO-MORBIDITIES AND TENDENCIES IN PATIENTS WITH NON-ISCHAEMIC HEART FAILURE (NIHF) - A LARGE OBSERVATIONAL COHORT STUDY SPANNING 14 YEARS}

${ }^{1} \mathrm{D}$ Rasoul*, ${ }^{2}$ Sam Wong, ${ }^{3}$ Suresh Chandran, ${ }^{3}$ Hardeep Uppal, ${ }^{4}$ Jaydeep Sarma, ${ }^{3}$ Rahul Potluri. 'The Royal Liverpool University Hospital; ${ }^{2}$ Macclesfield District General Hospital; ${ }^{3}$ ACALM Study Unit; ${ }^{4}$ University Hospital of South Manchester NHS Foundation Trust; *Presenting Author

\subsection{6/heartjnl-2016-309890.10}

Introduction Studies have shown that one in four psychiatric patients also suffer from a cardiovascular co-morbidity, and in recent years the links between cardiovascular disease and psychiatric conditions have been on the rise. We decided to investigate the prevalence of psychiatric co-morbidities and tendencies in patients with NIHF over a 14 year period.

Methods We compiled an anonymous database of adult patients diagnosed with NIHF across 7 hospitals in the North of England, UK, during 01/01/2000 to 31/03/2013. We analysed the data for prevalence of psychiatric co-morbidities such as; anxiety disorder, schizophrenia, depression etc. and for tendencies such as substance abuse and suicide attempts. We traced our patients with the ACALM (Algorithm for Co-morbidities, Associations, Length of Stay and Mortality) study protocol, which uses ICD-10 and OPCS-4 codes to allocate patients for statistical analysis using SPSS Version 20.0.

Results Between the years 2000-2013, 929,552 patients were admitted, $958(0.1 \%)$ of which had NIHF. The majority of our patients where male (68\%), had a mean age of 54.9 years \pm 13.9 years, and had a Caucasian background (77\%). Of these 958 patients, $8.1 \%(\mathrm{n}=78)$ had a psychiatric co-morbidity; depression and schizophrenia where most prevalent. Depression however, seems to more prevalent in dilated cardiomyopathy (DCM) as opposed to hypertrophic cardiomyopathy (HCM). 10.3\% ( $\mathrm{n}=99$ ) suffered from substance abuse, made up mainly by alcohol and tobacco abuse. 2 of our patients $(0.2 \%)$ where admitted following suicide attempts.

Conclusion It remains debatable whether these psychiatric conditions and tendencies represent a causative or correlative link. Studies exists to lend evidence to both claims. We did however find a steady increase in tobacco and alcohol abuse, and the diagnosis of depression in relation to our cohort during our 14-year period. We found that almost one in ten $(8.14 \%)$ patients with NIHF suffer from psychiatric co-morbidity and that one in ten $(10.33 \%)$ abuse some form of substance alongside their cardiac condition. Thus, a holistic approach to patients with NIHF should be adopted, and this approach would fall short if it did not explore patient's use of recreational drugs, suicidal intent and psychiatric wellbeing.

\section{THE DECENNIAL RELATIONSHIP BETWEEN HEART FAILURE AND ATRIAL FIBRILLATION - A LARGE POPULATION STUDY}

${ }^{1} \mathrm{D}$ Rasoul ${ }^{*},{ }^{2} \mathrm{~F}$ Ziaei, ${ }^{2} \mathrm{SKA}$ Shan, ${ }^{3} \mathrm{~J}$ Khan, ${ }^{2} \mathrm{R}$ Bhayani, ${ }^{2} \mathrm{~S}$ Shakir, ${ }^{4}$ Hardeep Uppal, ${ }^{5} \mathrm{~S}$ Chandran, ${ }^{6} \mathrm{~J}$ Sarma, ${ }^{4}$ Rahul Potluri. ${ }^{1}$ The Royal Liverpool University Hospital; ${ }^{2}$ Pennine Acute Hospitals NHS Trust; ${ }^{3}$ Pennine Acute Hospitals NHS Trust, Manchester, UK; ${ }^{4}$ ACALM Study Unit in Collaboration with Aston Medical School; ${ }^{5}$ North Western Deanery; ${ }^{6}$ University Hospital of South Manchester NHS Foundation Trust; *Presenting Author

\subsection{6/heartjnl-2016-309890.11}

Introduction Heart Failure (HF) is common in the western world, has multiple causes and confers significant morbidity and mortality. It is thought a significant proportion of HF patients also have atrial fibrillation (AF) although data from a large population in the UK are lacking. We studied the prevalence of $\mathrm{AF}$ in patients with $\mathrm{HF}$ in the North West of England.

Methods Anonymous data of adult patients aged $\geq 18$ with HF and AF attending seven hospitals between 2000-2013 was obtained and processed using the ACALM (Algorithm for Comorbidity, Associations, Length of stay and Mortality) study protocol. ACALM uses the ICD-10 and OPCS-4 coding systems to identify patients and the data was analysed in SPSS.

Results Of 929,465 adult patient population there were 31,760 (3.4\%) patients with HF. Mean age 73.6 years with a male population of $50.3 \%$. Of these 10,992 (34.6\%) patients had AF. The prevalence of AF in patients with $\mathrm{HF}$ increases with age from $8.1 \%$ in the $30-39$ year age group to $42.4 \%$ in the 80-89 years group (See Table 1).

Conclusion Whilst a significant number of elderly patients with HF patients do have concurrent AF, we found that the majority of patients do not. We believe our findings are 